\title{
Radiation Output Problem
}

National Cancer Institute

\section{Source}

National Cancer Institute. Radiation Output Problem. NCI Thesaurus. Code C133562.

Problem with the device's intended output of radiation. 